\title{
Autophagy impacts on oxaliplatin-induced hepatocarcinoma apoptosis via the IL-17/IL-17R-JAK2/STAT3 signaling pathway
}

\author{
JINGHUA WU ${ }^{1,2}$, JIAPEI GUO ${ }^{2}$, QING CAO $^{3}$, YI WANG ${ }^{2}$, \\ JUNMAO CHEN $^{2}$, ZHIGANG WANG ${ }^{4}$ and ZHIYONG YUAN ${ }^{1}$ \\ ${ }^{1}$ Key Laboratory of Cancer Prevention and Therapy, National Clinical Research Center for Cancer, \\ Tianjin Medical University Cancer Institute and Hospital, Tianjin 300000; ${ }^{2}$ Clinical Laboratory, \\ Affiliated Hospital of North China University of Science and Technology, Tangshan, Hebei 063000; \\ ${ }^{3}$ Clinical Laboratory, Hebei Medical University Second Hospital, Shijiazhuang, Hebei 050000; \\ ${ }^{4}$ Clinical Laboratory, Tangshan Fengrun Region Second People's Hospital, Tangshan, Hebei 063000, P.R. China
}

Received May 29, 2015; Accepted October 27, 2016

DOI: $10.3892 / \mathrm{ol} .2016 .5476$

\begin{abstract}
The interleukin (IL)-17/IL-17 receptor (IL-17R) complex has been shown to be important for the regulation of inflammation; however, its role in the regulation of tumor processes has recently emerged as a research focus. The present study demonstrated that oxaliplatin was able to increase the levels of IL-17/IL-17R in hepatocellular carcinoma (HCC) patients and cells lines, and that it had important roles in reducing the susceptibility of the cells to oxaliplatin-induced apoptosis. Furthermore, the expression of autophagy-related proteins was induced by IL-17/IL-17R and autophagy was shown to induce resistance to oxaliplatin in HCC. In addition, the janus kinase 2 (JAK2)/signal transducer and activator of transcription 3 (STAT3) pathway was shown to be an important pathway in the induction of autophagy in response to oxaliplatin. Autopjhagy was inhibited by 3 -methyladenine and JAK2/STAT3 signaling was blocked by AG490, which induced apoptosis in SMMC7721 cells treated with oxaliplatin. The results of the present study may help to elucidate the mechanism underlying the role of IL-17/IL-17R-induced autophagy in the chemoresistance of HCC, as well as help to establish and develop measures to overcome chemoresistance in HCC.
\end{abstract}

\section{Introduction}

Hepatocellular carcinoma (HCC) is a major malignancy worldwide and its incidence is increasing annually; it is the second most common cause of cancer-associated mortality (1).

Correspondence to: Professor Zhiyong Yuan, Key Laboratory of Cancer Prevention and Therapy, National Clinical Research Center for Cancer, Tianjin Medical University Cancer Institute and Hospital, Huanhu West Road, Tianjin 300000, P.R. China

E-mail: tswujinghua@163.com

Key words: hepatocellular carcinoma, interleukin-17/interleukin-17 receptor, autophagy, janus kinase 2/signal transducer and activator of transcription 3
The majority of patients have a low survival rate as a result of locally advanced or metastatic diseases, and surgery is feasible for only a small percentage of patients with HCC. Therefore, chemotherapy is the optimal therapeutic strategy for inoperable HCC (2). Oxaliplatin has been widely used in chemotherapy to reduce tumor recurrence and prolong survival in patients with HCC because of its fewer side effects compared with other platinum drugs (3). However, chemoresistance to oxaliplatin in the form of suppressed HCC apoptosis is commonly observed (4).

Interleukin-17 (IL-17) is predominantly secreted by interleukin-17-producing T-helper (Th17) cells, which participate in the progression and pathogenesis of inflammatory diseases (3). The IL-17 receptor (IL-17R) is expressed on the surface of numerous cells, including macrophages, dendritic cells, epithelial cells, fibroblasts and T lymphocytes $(5,6)$. Previous studies reported that IL-17-producing cells accumulated in tumors $(7,8)$, and that patients with malignant serum effusions (9) or multiple myeloma (10) showed significantly higher serum levels of IL-17. Furthermore, patients with persistently higher levels of IL-17 demonstrated the requirement for longer courses of chemotherapy, since these patients comprised a significant proportion of all cases of recurrence (11). Typically, IL-17 does not engage with Toll/IL-1 receptor (TIR) domain-containing adaptors, such as MyD88, TIR domain-containing adapter protein inducing interferon- $\beta$ or IL-1 receptor-associated kinases (12). Rather, IL-17 signals through nuclear factor (NF) $\kappa \mathrm{B}$ (13), mitogen-activated protein kinase (MAPK) (14) and phosphoinositide 3-kinase (PI3K) (14) signaling pathways. The janus kinase 2 (JAK2)/signal transducer and activator of transcription 3 (STAT3) signaling pathway plays an important role in regulating a number of pathways associated with tumorigenesis, including cell cycle progression, apoptosis and tumor cell evasion of the immune system $(15,16)$. In a previous study, phosphorylation of STAT3 was markedly increased as early as $3 \mathrm{~h}$ following IL-17 treatment and lasted for $24 \mathrm{~h}$ (17), which indicated that JAK2/STAT3 signaling may have important roles in tumor progression associated with IL-17.

Autophagy involves lysosomal-mediated degradation of cellular organelles and has been closely related to tumor 
occurrence and progression (18). A previous study reported that resistance to oxaliplatin in HepG2 cells could be recovered by inhibition of autophagy (19), which suggested opportunities for exploitation of autophagy as a therapeutic target in cancer.

Although the association between IL-17 and tumour chemotherapy has been previously investigated (20), the underlying mechanism remains unclear. Therefore, the present study aimed to elucidate the role of IL-17/IL-17R-induced autophagy in the resistance of HCC cells to oxaliplatin, and to determine the potential underlying mechanism.

\section{Materials and methods}

Patient samples and tissue processing. A series of HCC specimens were obtained from 30 patients with pathologically confirmed HCC at the Affiliated Hospital of North China University of Science and Technology (Tangshan, China). No patients received adjuvant chemotherapy, radiotherapy or surgery prior to admission. All patients were administered one course ( 2 weeks) of oxaliplatin, after which the concentration of IL-17 in sera and IL-17R mRNA levels were detected. In addition, matched normal hepatic tissues were obtained from 28 patients who were admitted to hospital due to wounds obtained in a fall or traffic accident. Peripheral blood samples ( $3 \mathrm{ml}$ ) were collected from all patients. HCC biopsy specimens for the detection of IL-17R mRNA expression levels by reverse transcription-quantitative polymerase chain reaction (RT-qPCR) were collected via paracentesis per cutem prior to and following oxaliplatin treatment or surgical resection. Serum IL-17 levels were determined using an ELISA. The present study was approved by the Institutional Review Board of North China University of Science and Technology. Informed consent was obtained from all patients prior to specimen collection.

Cell lines and culture conditions. The human SMMC-7721, L02 and HepG2 cells lines were maintained at $37^{\circ} \mathrm{C}$ in a humidified atmosphere containing $5 \% \mathrm{CO}_{2}$ in high-glucose Dulbecco's modified Eagle's medium (Gibco; Thermo Fisher Scientific, Inc., Waltham, MA, USA) supplemented with $10 \%$ heat-inactivated fetal bovine serum (HyClone; GE Healthcare Life Sciences, Logan, UT, USA), 100 units/ml penicillin and $100 \mathrm{mg} / \mathrm{ml}$ streptomycin.

ELISA assays. Serum levels of IL-17 (pg/ml) were measured using a solid phase sandwich ELISA assay according to the manufacturer's protocols (R\&D Systems, Inc., Minneapolis, MN, USA).

$R N A$ isolation and RT-qPCR. To examine IL-17R expression in HCC patients prior to and following oxaliplatin therapy, IL-17R mRNA expression levels in the tumor tissues were compared with matched normal tissues by RT-qPCR. Total RNA was extracted and reverse transcribed using an RNeasy kit (Thermo Fisher Scientific, Inc., Pittsburgh, PA, USA), according to the manufacturer's protocol. RNA (1 $\mu \mathrm{g})$, along with $10 \mathrm{X}$ DNase I reaction buffer and $1 \mu \mathrm{g}$ DNase I RNase-free was transferred to a $1.5 \mathrm{ml}$ tube where the volume was adjusted to $10 \mu \mathrm{l}$ using RNase-free water. After incubating for $30 \mathrm{~min}$ at $37^{\circ} \mathrm{C}$, the DNase I was inactivated by the addition of $1 \mu 125 \mathrm{mM}$ EDTA. The mixture was subsequently heated for $10 \mathrm{~min}$ at $65^{\circ} \mathrm{C}$. qPCR was performed in $20 \mu 1$ reaction volumes containing $2.0 \mu \mathrm{l} \mathrm{cDNA}, 0.4 \mu \mathrm{l}$ of each primer, $6.0 \mu \mathrm{l}$ $\mathrm{ddH}_{2} \mathrm{O}, 0.4 \mu \mathrm{l}$ ROX reference dye and $10 \mu \mathrm{l}$ fluorescent SYBR Green (Takara Bio, Inc., Otsu, Japan). Amplification was performed in 96-well optical plates on a 7300 Real-Time PCR system (Applied Biosystems; Thermo Fisher Scientific, Inc., Waltham, MA, USA) with $30 \mathrm{sec}$ incubation at $95^{\circ} \mathrm{C}$, followed by 45 cycles of $95^{\circ} \mathrm{C}$ for $5 \mathrm{sec}$ and $60^{\circ} \mathrm{C}$ for $60 \mathrm{sec}$. The primers used were as follows: IL-17R forward, 5'-CACTCA CTCTACGCAACCTTAA-3', reverse, 5'-AGATGCCCGTGA TGAACC-3'; and GAPDH forward, 5'-GCACCGTCAAGG CTGAGAAC-3' and reverse, 5'-ATGGTGGTGAAGACGCCA GT-3'.

Each sample was analyzed in triplicate. The $2^{-\Delta \Delta \mathrm{Cq}}$ method of relative quantification was performed to calculate relative changes in the mRNA expression levels of target genes.

Cytokine inhibitor treatment. Cells were cultured in 6-well plates and treated with $20 \mu \mathrm{g} / \mathrm{ml} \mathrm{Oxa}$ in the presence or absence of an anti-IL-17R antibody $(10 \mu \mathrm{g} / \mathrm{ml})$ or IL-17 $(200 \mathrm{ng} / \mathrm{ml})$ for $18 \mathrm{~h}$. The apoptosis-related proteins BCL-2 and BAX were measured by western blotting. LY294002 (Beyotime Institute of Biotechnology, Haimen, China), a PI3K-specific inhibitor, AG490 (Beyotime Institute of Biotechnology), a JAK2 inhibitor, and 3-MA (Sigma-Aldrich; Merck Millipore, Darmstadt, Germany), an inhibitor of autophagy, were dissolved in dimethyl sulfoxide prior to use. These inhibitors were added to the culture medium $1 \mathrm{~h}$ prior to oxaliplatin treatment, with AG490 added at a dose of $15 \mu \mathrm{g} / \mathrm{ml}$ and LY294002 at $7.5 \mu \mathrm{g} / \mathrm{ml}$. Cells were treated with $20 \mu \mathrm{g} / \mathrm{ml}$ oxaliplatin for $18 \mathrm{~h}$. No cell cytotoxicity of these inhibitors, as assessed using a nuclear dye exclusion assay (21), was observed at the doses used in this study (data not shown).

Western blotting. Following treatment of the cells with oxaliplatin $(20 \mu \mathrm{g} / \mathrm{ml})$, the cells were lysed in whole-cell lysate (Wuhan Boster Biological Technology, Ltd., Wuhan, China) containing phenylmethylsulfonyl fluoride and a phosphatase inhibitor. Equal quantities of cell lysate $(60 \mu \mathrm{g})$ were separated by $10 \%$ SDS-PAGE and transferred onto polyvinylidene difluoride membranes. After blocking in $5 \%$ evaporated milk for $1 \mathrm{~h}$ at $37^{\circ} \mathrm{C}$, the membranes were incubated with the following primary antibodies: Anti-IL-17R (\#D1Y4C), anti-B-cell lymphoma (BCL)-2 (\#D55G8), anti-BCL-2-associated X protein (BAX; \#D2E11), anti-microtubule-associated protein 1 light chain $3 \beta$ (LC3B; \#D11), anti-JAK2 (\#D2E12), anti-phosphorylated (p)-JAK2 (\#D15E2), anti-STAT3 (\#D3Z2G), anti-p-STAT3 (\#6E4) (all 1:1,000 dilution; Cell Signaling Technology, Inc., Danvers, MA, USA) and anti-Beclin-1 (dilution, 1:500; \#B6061; Sigma-Aldrich; Merck Millipore). GAPDH was used as a loading control and was detected using an anti-GAPDH antibody (dilution, 1:5,000; \#AP0066; Bioworld Technology, Inc., St. Louis Park, MN, USA). The membrane was incubated for $1 \mathrm{~h}$ at $37^{\circ} \mathrm{C}$ with goat anti-mouse immunoglobulin (Ig)G (dilution, 1:10,000; \#ab6785) and goat anti-rabbit IgG (dilution, 1:10,000; \#ab6721) (Abcam, Cambridge, UK). After washing the membrane for $45 \mathrm{~min}$ with cleaning solution, proteins were detected using an enhanced chemiluminescence 
A

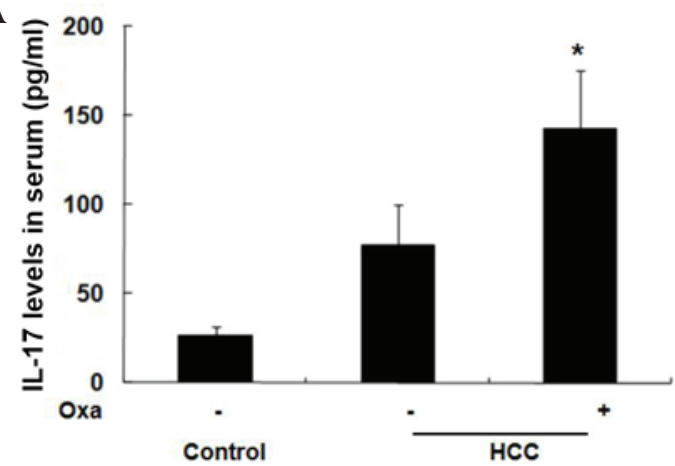

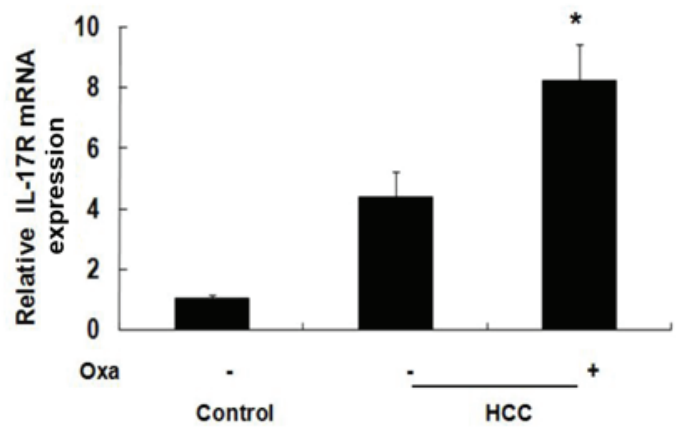

Figure 1. IL-17 and IL-17R expression in HCC patients. (A) Serum samples from 30 HCC patients prior to and following Oxa treatment, as well as 30 samples from healthy controls, were assayed using an IL-17 ELISA. (B) Tissues samples from 30 HCC patients prior to and following Oxa treatment, as well as 30 samples from controls, were assayed by reverse transcription-quantitative polymerase chain reaction. * $\mathrm{P}<0.05$ vs. Oxa-untreated group. IL-17, interleukin-17; IL-17R, IL-17 receptor; HCC, hepatocellular carcinoma; Oxa, oxaliplatin.

A

\section{L02 HepG2 SMMC7721}

Anti-IL-17R

Anti-GAPDH

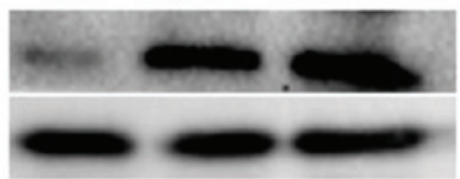

B

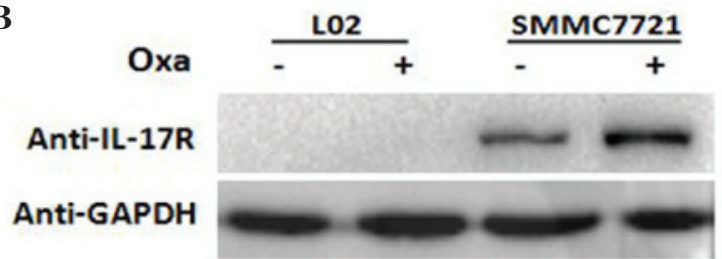

Figure 2. Expression of IL-17R in hepatocarcinoma cells. (A) IL-17R expression L02, HepG2 and SMMC-7721 cells was detected by western blot analysis. (B) After culturing L02 and SMMC-7721 cells for $18 \mathrm{~h}$ in the presence of $20 \mu \mathrm{g} / \mathrm{ml}$ Oxa, the expression of IL-17R was measured by western blotting. GAPDH was used as an internal control. IL-17R, interleukin-17 receptor; Oxa, oxaliplatin.

system and graphs were analyzed using Image Lab software v2.5.1 (Bio-Rad Laboratories, Inc., Hercules, CA, USA). Each experiment was repeated three times.

Statistical analysis. Statistical analyses were performed using SPSS 17.0 software (SPSS, Inc., Chicago, IL, USA). Graphs were analyzed using the Image Lab system. Data are expressed as the mean \pm standard deviation of the values from three independent experiments. Statistical analyses were conducted using either the Student's t-test or one-way analysis of variance in comparison with corresponding controls. $\mathrm{P}<0.05$ was considered to indicate a statistically significant difference.

\section{Results}

Expression of IL-17 and IL-17R in HCC patients. IL-17 has a role in numerous autoimmune and inflammatory conditions, including rheumatoid arthritis, multiple sclerosis, psoriasis, Crohn's disease and systemic lupus erythematosus, through combining with IL-17R (22). However, evidence has shown that IL-17 may also contribute to disease progression and treatment response in patients with tumors (23). In the present study, the levels of IL-17 were significantly increased in oxaliplatin-treated HCC patients, as compared with untreated HCC patients. Prior to treatment, the serum levels of IL-17 were $77.36 \pm 22.90 \mathrm{pg} / \mathrm{ml}$, but were significantly increased up to $142.41 \pm 33.25 \mathrm{pg} / \mathrm{ml}$ after one course of treatment ( 2 weeks) $(\mathrm{P}<0.05$; Fig. 1A). Furthermore, the mRNA expression levels of IL-17R in oxaliplatin-treated HCC biopsy specimens were significantly higher compared with untreated HCC biopsy specimens $(\mathrm{P}<0.05$; Fig. 1B). These results suggest that oxaliplatin increases the expression of

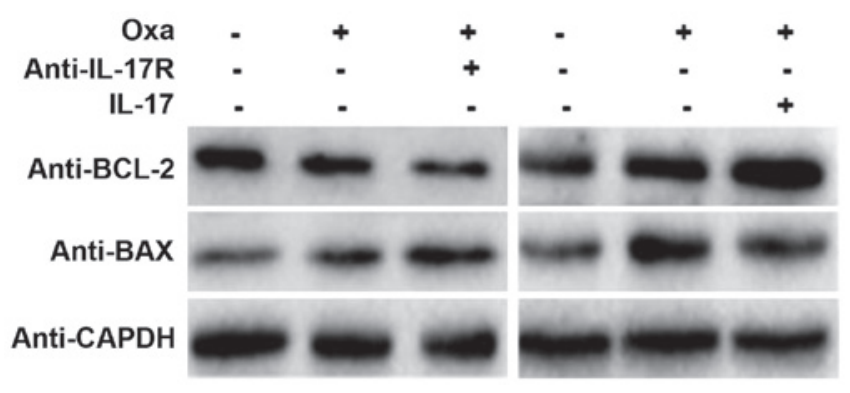

Figure 3. Effect of IL-17/IL-17R on the apoptosis of SMMC-7721 cells SMMC-7721 cells were treated with $20 \mu \mathrm{g} / \mathrm{ml}$ Oxa in the presence or absence of an anti-IL-17R antibody $(10 \mu \mathrm{g} / \mathrm{ml})$ or IL-17 $(200 \mathrm{ng} / \mathrm{ml})$ for $18 \mathrm{~h}$. The apoptosis-related proteins BCL-2 and BAX were measured by western blotting. GAPDH was used as an internal control. Oxa, oxaliplatin; IL-17, interleukin-17; IL-17R, IL-17 receptor; BCL-2, B-cell lymphoma-2; BAX, BCL-2-associated X protein.

IL-17/IL-17R in patients with HCC, and that there is an association between oxaliplatin-induced apoptosis and IL-17/IL-17R.

Oxaliplatin induces the expression of IL-17R in HCC cells. L02, HepG2 and SMMC-7721 cell lines were cultured in the presence of oxaliplatin and western blot analysis was performed to measure the amount of IL-17R protein in each cell line. The results showed that IL-17R was expressed in HepG2 and SMMC-7721 cells, but not in L02 cells (Fig. 2A).

In order to confirm the changes in IL-17R expression following oxaliplatin treatment of SMMC-7721 cells, oxaliplatin $(20 \mu \mathrm{g} / \mathrm{ml})$ was added to SMMC-7721 and L02 cells for $18 \mathrm{~h}$. Western blotting showed that the expression of IL-17R 
A

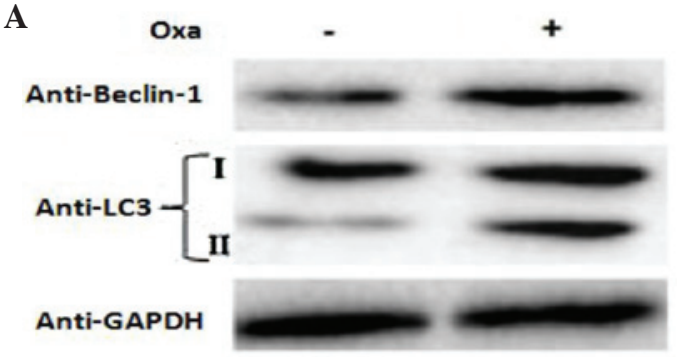

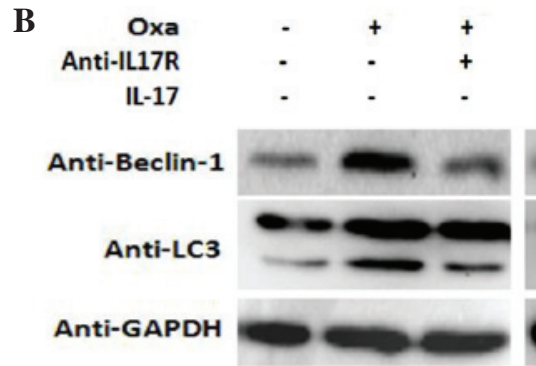

C

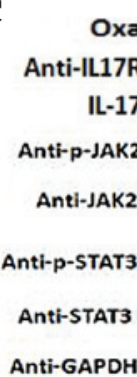

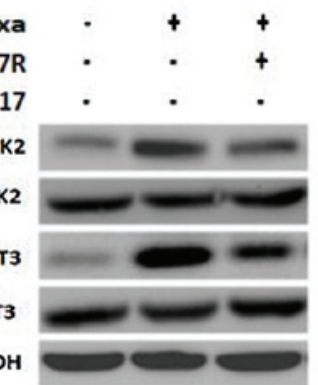

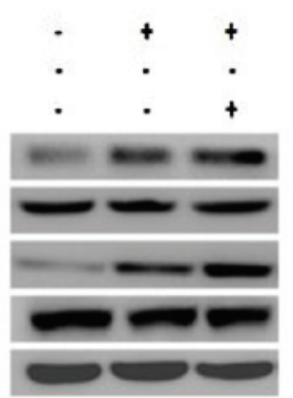

D

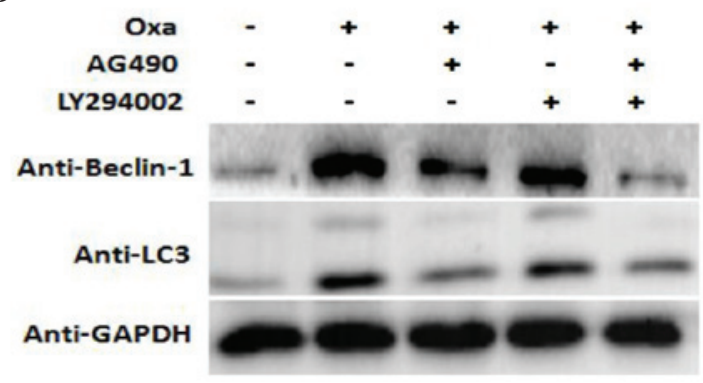

Figure 4. IL-17/IL-17R induces autophagy via the JAK2/STAT3 signaling pathway. SMMC-7721 cells were treated with $20 \mu \mathrm{g} / \mathrm{ml}$ Oxa in the presence or absence of (A) anti-IL-17R (10 $\mu \mathrm{g} / \mathrm{ml})$ or (B) IL-17 $(200 \mathrm{ng} / \mathrm{ml})$ for $18 \mathrm{~h}$. (C) The activation of JAK2 and STAT3 was detected by western blotting. (D) LY294002 $(10 \mu \mathrm{g} / \mathrm{ml})$ and AG490 $(15 \mu \mathrm{g} / \mathrm{ml})$ were added to the culture medium for $1 \mathrm{~h}$ prior to Oxa treatment. LC3 and Beclin-1 protein expression was determined by western blot analysis. GAPDH was used as an internal control. Oxa, oxaliplatin; IL-17, interleukin-17; IL-17R, IL-17 receptor; JAK2, janus kinase 2; STAT3, signal transducer and activator of transcription 3; LC3, microtubule-associated protein 1 light chain.

increased markedly in oxaliplatin-treated SMMC-7721 cells compared with the untreated cells (Fig. 2B). These results suggest that IL-17 is expressed in HCC cells and is increased following oxaliplatin treatment.

IL-17/IL-17R inhibits oxaliplatin-induced apoptosis of SMMC-7721 cells. As shown in Fig. 2, IL-17R was expressed in SMMC-7721 cells. Furthermore, expression of IL-17R in SMMC-7721 cells increased markedly following oxaliplatin treatment. However, the role of IL-17R oxaliplatin-induced in these cells is unknown. As a mechanism for programmed cell death, apoptosis is regulated by the BCL-2 family proteins, including BAX, which control the sensitivity of cells to apoptotic stresses. BCL-2 is an anti-apoptosis gene, whereas BAX is an apoptosis-promoting matrix gene (24). In the present study, IL-17R-blocking and IL-17-promoting assays were designed to observe the effect of IL-17/IL-17R on oxaliplatin-induced apoptosis. Western blotting showed that BCL-2 protein expression was decreased and BAX protein expression was increased in oxaliplatin-treated SMMC-7721 cells, as compared with the untreated cells, which indicated that apoptosis was induced by oxaliplatin treatment (Fig. 3). When the IL-17R on the surface of SMMC-7721 cells was blocked by a neutralizing anti-IL-17R antibody (10 $\mu \mathrm{g} / \mathrm{ml}$; \#MAB177; R\&D Systems, Inc.), the expression of BCL-2 was decreased and that of BAX was increased (Fig. 3). Conversely, the expression of BCL-2 was increased and that of BAX was decreased in IL-17-promoting SMMC7721 cells. These results suggest that IL-17/IL-17R may inhibit HCC apoptosis and that blocking IL-17R is able to upregulate the susceptibility of HCC cells to oxaliplatin-induced apoptosis.

IL-17/IL-17R induces autophagy in SMMC-7721 cells. Increasingly, studies have focused on the effect of autophagy on tumor

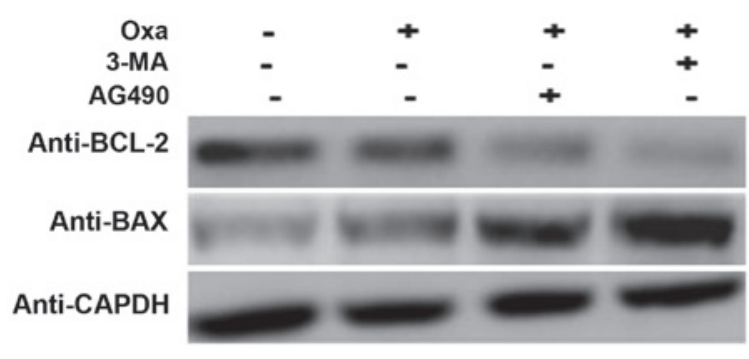

Figure 5. IL-17/IL-17R downregulate apoptosis by inducing autophagy. 3-MA $(10 \mathrm{mM})$ and AG490 $(15 \mu \mathrm{g} / \mathrm{ml})$ were added to the culture medium for $1 \mathrm{~h}$ prior to Oxa treatment, after which SMMC-7721 cells were treated with $20 \mu \mathrm{g} / \mathrm{ml}$ Oxa for $18 \mathrm{~h}$. BCL-2 and BAX protein expression was determined by western blot analysis. GAPDH was used as an internal control. Oxa, oxaliplatin; IL-17, interleukin-17; IL-17R, IL-17 receptor; BCL-2, B-cell lymphoma-2; BAX, BCL-2-associated X protein; 3-MA, 3-methyladenine.

progression $(25,26)$. Autophagy is a reversible process that regulates tumor survival or death; thus, it is closely associated with tumor progression (27). Furthermore, autophagy during chemotherapy has been shown to induce chemoresistance (28), while sensitivity to chemotherapy was increased when autophagy was inhibited $(29,30)$.

It is well known that decreased susceptibility to apoptosis during chemotherapy is the main mechanism of chemoresistance $(31,32)$. LC3 is a specific marker of autophagosomes in mammalian cells, and the conversion of the soluble form of LC3 (LC3-I) to the autophagosome-associated form (LC3-II) is a characteristic of autophagy (33-35). Furthermore, Beclin-1 is another important autophagy gene. In the present study, Beclin-1 and LC3-II expression in SMMC-7721 cells was increased following treatment with oxaliplatin (Fig. 4A). In addition, the expression of Beclin-1 and LC3-II was markedly attenuated following blocking of IL-17R with a neutralizing 
antibody, and was increased accordingly following exposure to IL-17 (Fig. 4B).

IL-17/IL-17R induces autophagy in SMMC-7721 cells through the JAK2/STAT3 signaling pathway. IL-17 mediates cellular activities through various signal transduction pathways, including NF- $\kappa \mathrm{B}, \mathrm{MAPK}$ and PI3K (14). The present study investigated the potential role of the JAK2/STAT3 signaling pathway in IL-17-mediated autophagy. In the present study, the levels of p-JAK2 and p-STAT3 were increased in oxaliplatin-stimulated cells compared with the control cells, while they were decreased following blocking of IL-17R. Conversely, treatment with IL-17 (Sigma-Aldrich; Merck Millipore) increased the levels of p-JAK2 and p-STAT3 in SMMC-7721 cells (Fig. 4C). These results suggest that IL-17/IL-17R mediate cellular responses through the JAK2/STAT3 signaling pathway.

In order to investigate the relationship between IL17/IL17R and JAK2/STAT3 and oxaliplatin-induced autophagy, blocking experiments were performed. AG490, which is a JAK inhibitor, is able to specifically block the activation of the JAK2/STAT3 signaling pathway, as tyrosine phosphorylation of STAT3 is dependent on JAK activity (36). Furthermore, since the PI3K pathway has a crucial role in autophagy (37-39), the PI3K signaling pathway was used as a control pathway to investigate the JAK2/STAT3 pathway. LY294002, which is reported to inhibit AKT activation in a dose-dependent manner (40), was used and compared with the effect of AG490. SMMC-7721 cells were incubated with AG490 (15 $\mu \mathrm{g} / \mathrm{ml})$ or LY294002 $(10 \mu \mathrm{g} / \mathrm{ml})$ for $1 \mathrm{~h}$ prior to oxaliplatin treatment, after which autophagy-related proteins were detected by western blotting. Notably, a marked decrease in the expression of both Beclin-1 and LC3 II proteins was observed in AG490-treated cells, while only a negligible change was observed in LY294002-treated cells (Fig. 4D). These results suggest that inhibition of the JAK2/STAT3 signaling pathway suppresses oxaliplatin-induced autophagy to a greater extent than inhibition of the PI3K pathway, which further indicates that the JAK2/STAT3 signaling pathway may have an important role in oxaliplatin-induced autophagy.

In order to further confirm the role of IL-17/IL-17R and the JAK2/STAT3 signaling pathway in the induction of autophagy and inhibition of apoptosis, SMMC-7721 cells were treated with 3-methyladenine (3-MA; 10 mM; Sigma-Aldrich; Merck Millipore), which is a known inhibitor of autophagy (41), and AG490 to inhibit the JAK2/STAT3 signaling pathway. Compared with the cells treated with oxaliplatin only, the AG490- and oxaliplatin-treated cells expressed less BCL-2 and more BAX, which indicated that apoptosis was induced to a greater extent in these cells. There was no difference in the expression of BCL-2 and BAX following treatment with AG490 and 3-MA (Fig. 5). These results suggest that autophagy in oxaliplatin-treated cells is induced through the JAK2/STAT3 signaling pathway.

Together, the results of the present study suggest that autophagy is activated in HCC cells following oxaliplatin treatment via IL17/IL17R-JAK2/STAT3, and that this may be involved in the chemoresistance of HCC to oxaliplatin.

\section{Discussion}

IL-17, which is the hallmark cytokine of the newly-defined Th17 cell subset, serves an important role in inflammatory diseases (42). Since chronic inflammation has been associated with tumor invasion, migration and metastasis (43), the significance of IL-17 in tumor progression has received increasing attention. The receptor of IL-17, IL-17R, is a common signaling subunit used by multiple ligands (44). Researches have begun to investigate the unusual functional motifs and novel proximal signaling mediators employed by the IL-17R family to mediate downstream events $(7,45)$. There is evidence that IL-17 may emerge as a novel prognostic marker in HCC.

In the present study, it was demonstrated that autophagy downregulated oxaliplatin-induced HCC apoptosis through the IL-17/IL-17R-JAK2/STAT3 signaling pathway. IL-17 and IL-17R were markedly increased in oxaliplatin-treated HCC patients. Previous studies reported that IL-17 may exert pro-tumor or antitumor effects in various tumor contexts (46-48). The explanation for this discrepancy remains unknown. The present study aimed to determine the role that IL-17/IL-17R plays in the response of HCC to chemotherapy. Western blot analysis demonstrated that the expression of IL-17R was upregulated in HepG2 and SMMC-7721 cell lines, which was consistent with the report that IL-17-producing cells accumulate in various cancers, including HCC (8). In addition, IL-17/IL-17R expression was shown to increase in oxaliplatin-treated SMMC-7721 cells, which was associated with downregulation of apoptosis, as demonstrated by the detection of BCL-2 and BAX expression by western blotting.

Previous studies have focused on the relationship between autophagy and tumors $(49,50)$. The role of autophagy in tumors is complex, as it has been associated with both tumor suppression and therapeutic resistance in advanced tumors (51). Inhibition of autophagy acted synergistically with chemotherapy in a mouse model of lymphoma (52). Autophagy was considered a temporary survival mechanism through the interaction of autophagy-related Beclin-1 and anti-apoptotic BCL-2 (53); thus, autophagy may be induced upon chemotherapy as a survival mechanism. In the present study, the reduced sensitivity of HCC cells to apoptosis may have been related to the induction of autophagy. Therefore, the expression of autophagy-related proteins, including LC3B and Beclin-1, was detected in oxaliplatin-treated SMMC-7721 cells. It was determined that oxaliplatin was able to induce autophagy in HCC and that autophagy was dependent on IL-17/IL-17R. To delineate the potential mechanism underlying IL-17/IL-17R-induced autophagy, the activation status of JAK2 and STAT3 under the interference of IL-17/IL-17R was observed. It was found that IL-17/IL-17R increased the phosphorylation of JAK2 and STAT3, which was consistent with the fact that STAT3 controls Th17 cell differentiation (54). Previous studies have shown that the PI3K signaling pathway has a crucial role in autophagy (55-57). Therefore, to confirm the roles of the PI3K and/or the JAK2/STAT3 signaling pathway in autophagy activation in oxaliplatin-treated HCC cells, SMMC-7721 cells were pre-treated with both LY294002 and AG490 to block PI3K and JAK2/STAT3. According to the western blot result, autophagy was inhibited to a greater extent in AG490-treated cells, which suggested that the JAK2/STAT3 signaling pathway was predominantly involved. 3-MA is a specific autophagy inhibitor, and pre-treatment of SMMC-7721 cells with 3-MA induced apoptosis to a greater extent than treatment with oxaliplatin alone by increasing BAX 
and decreasing BCL-2 expression. Similarly, cells treated with AG490 expressed less BCL-2 and more BAX, thus confirming that autophagy was induced by the JAK2/STAT3 signaling pathway.

Consistent with the results of the present study, Huang et al (58) reported that a JAK inhibitor was able to effectively suppress IL-17A-induced gene expression in human bronchial epithelial cells. Sun et al (59) suggested that STAT3 signaling was a key pathway that mediates immune suppression in the tumor microenvironment, and that aberrantly activated STAT3 in HCC cells resulted in the upregulation of cytokines, including IL-17. Cross-Knorr et al (60) showed that oxaliplatin was able to enhance the apoptosis of cancer cells by disrupting survival signaling via the JAK/STAT pathway at the receptor level in stage II colon cancer patients. Together, these findings suggested the importance of the JAK2/STAT3 signaling in the regulation of IL-17 signaling.

Previous reports have also indicated that pharmacological inhibitors of JAKs are able to limit IL-17 signaling (61), although these results should be interpreted with caution because of the non-specific effects of such compounds (62). Furthermore, IL-17-induced activation of STAT factors, which could promote cytokines secretion (including IL-6), have not been satisfactorily disproved (7). Therefore, whether IL-17/IL-17R mediates oxaliplatin-induced autophagy directly or through other pathways requires further analysis.

In conclusion, the present study demonstrated that autophagy inhibited oxaliplatin-induced HCC apoptosis via the IL-17/IL-17R-JAK2/STAT3 signaling pathway. These results suggested that blocking IL-17/IL-17R may be considered a novel therapy for chemoresistant HCCs.

\section{Acknowledgements}

This study was supported by the Natural Science Foundation of Hebei Province (grant no. 2016209007).

\section{References}

1. Zhao Y, Wang Q, Deng X, Shi P and Wang Z: Quantitative assessment of the association between GSTP1 gene Ile105Val polymorphism and susceptibility to hepatocellular carcinoma. Tumor Biol 34: 2121-2126, 2013.

2. Louaf S, Boige V, Ducreux M, Bonyhay L, Mansourbakht T, de Baere T, Asnacios A, Hannoun L, Poynard T and Taïeb J: Gemcitabine plus oxaliplatin (GEMOX) in patients with advanced hepatocellular carcinoma (HCC): Results of a phase II study. Cancer 109: 1384-1390, 2007.

3. Sun HQ, Zhang JY, Zhang H, Zou ZS, Wang FS and Jia JH: Increased Th17 cells contribute to disease progression in patients with HBV-associated liver cirrhosis. J Viral Hepat 19: 396-403, 2012.

4. Du H, Yang W, Chen L, Shi M, Seewoo V, Wang J, Lin A, Liu Z and Qiu W: Role of autophagy in resistance to oxaliplatin in hepatocellular carcinoma cells. Oncol Rep 27: 143-150, 2012.

5. Shen F and Gaffen SL: Structure-function relationships in the IL-17 receptor: Implications for signal transduction and therapy. Cytokine 41: 92-104, 2008.

6. Gaffen SL: Recent advances in the IL-17 cytokine family. Curr Opin Immunol 23: 613-619, 2011.

7. Ma Y, Aymeric L, Locher C, Mattarollo SR, Delahaye NF, Pereira P, Boucontet L, Apetoh L, Ghiringhelli F, Casares N, et al: Contribution of IL-17-producing gamma delta T cells to the ecacy of anticancer chemotherapy. J Exp Med 208: 491-503, 2011.

8. Lv L, Pan K, Li XD, She KL, Zhao JJ, Wang W, Chen JG Chen YB, Yun JP and Xia JC: The accumulation and prognosis value of tumor infiltrating IL-17 producing cells in esophageal squamous cell carcinoma. PLoS One 6: e18219, 2011.
9. Li Q, Han Y, Fei G, Guo Z, Ren T and Liu Z: IL-17 promoted metastasis of non-small-cell lung cancer cells. Immunol Lett 148: 144-150, 2012.

10. Lemancewicz D, Bolkun L, Jablonska E, Czeczuga-Semeniuk E, Kostur A, Kloczko J and Dzieciol J: The role of interleukin-17A and interleukin-17E in multiple myeloma patients. Med Sci Monit 18: BR54-BR59, 2012.

11. Droeser RA, Güth U, Eppenberger-Castori S, Stadlmann S, Hirt C, Terracciano L and Singer G: High IL-17-positive tumor immune cell infiltretion is indicative for chemosensitivity of ovarian carcinoma. J Cancer Res Clin Oncol 139: 1295-1302, 2013.

12. Maitra A, Shen F, Hanel W, Mossman K, Tocker J, Swart D and Gaffen SL: Distinct functional motifs within the IL-17 receptor regulate signal transduction and target gene expression. Proc Nat Acad Sci USA 104: 7506-7511, 2007.

13. Korn T: Th17 cells: Effector T cells with inflammatory properties. Semin Immunol 19: 362-371, 2007.

14. Gaffen SL: Structure and signaling in the IL-17 receptor family. Nat Rev Immunol 9: 556-567, 2009.

15. Okinaga T, Ariyoshi W and Akifusa S: Essential role of JAK/STAT pathway in the induction of cell cycle arrest in macrophages infected with periodontopathic bacterium Aggregatibacter actinomycetemcomitans. Med Microbiol Immunol 202: 167-74, 2013

16. Chen Y, Jiao B, Yao M, Shi X, Zheng Z, Li S and Chen L: ISG12a inhibits HCV replication and potentiates the anti-HCV activity of IFN- $\alpha$ through activation of the Jak/STAT signaling pathway independent of autophagy and apoptosis. Virus Res 10: S0168-S01702, 2016

17. Gu FM, Li QL, Gao Q, Jiang JH, Zhu K, Huang XY, Pan JF, Yan J, Hu JH, Wang Z, et al: IL-17 induces AKT-dependent IL-6/JAK2/STAT3 activation and tumor progression in hepatocellular carcinoma. Mol Cancer 10: 150, 2011.

18. Xu Y, An Y, Wang Y, Zhang C, Zhang H, Huang C, Jiang H, Wang $X$ and Li X: miR-101 inhibits autophagy and enhances cisplatin-induced apoptosis in hepatocellular carcinoma cells. Oncol Rep 29: 2019-2024, 2013.

19. Harris SJ, Ciuclan L, Finan PM, Wymann MP, Walker C, Westwick J, Ward SG and Thomas MJ: Genetic ablation of PI3K $\gamma$ results in defective IL-17RA signalling in T lymphocytes and increased IL-17 levels. Eur J Immunol 42: 3394-3404, 2012.

20. Murugaiyan G and Saha B: Protumor vs antitumor functions of IL-17. J Immunol 183: 4169-4175, 2009.

21. Fortmüller K, Alt K, Gierschner D, Wolf P, Baum V, Freudenberg N, Wetterauer U, Elsässer-Beile U and Bühler P: Effective targeting of prostate cancer by lymphocytes redirected by a PSMAxCD3 bispecific single-chain diabody. Prostate 71: 588-596, 2011.

22. Tesmer LA, Lundy SK, Sarkar S and Fox DA: Th17 cells in human disease. Immunol Rev 223: 87-113, 2008.

23. Martin F, Apetoh L and Ghiringhelli F: Controversies on the role of Th17 in cancer: A TGF- $\beta$-dependent immunosuppressive activity?. Trends Mol Med 18: 742-749, 2012.

24. Anilkumar U and Prehn JHM: Anti-apoptotic BCL-2 family proteins in acute neural injury. Front Cell Neurosci 30: 281, 2014.

25. Xu Y and Lv SX: The effect of JAK2 knockout on inhibition of liver tumor growth by inducing apoptosis, autophagy and anti-proliferation via STATs and PI3K/AKT signaling pathways. Biomed Pharmacother 84: 1202-1212, 2016.

26. Tan YQ, Zhang J and Zhou G: Autophagy and its implication in human oral diseases. Autophagy 20: 0, 2016.

27. Cui J, Gong Z and Shen HM: The role of autophagy in liver cancer: Molecular mechanisms and potential therapeutic targets. Biochim Biophys Acta 1836: 15-26, 2013.

28. Liu D, Yang Y, Liu Q and Wang J: Inhibition of autophagy by 3-MA potentiates cisplatin-induced apoptosis in esophageal squamous cell carcinoma cells. Med Oncol 28: 105-111, 2011.

29. Golden EB, Cho HY, Jahanian A, Hofman FM, Louie SG, Schönthal AH and Chen TC: Chloroquine enhances temozolomide cytotoxicity in malignant gliomas by blocking autophagy. Neurosurg Focus 37: E12, 2014.

30. Wu W, Li W, Zhou Y and Zhang C: Inhibition of beclin1 affects the chemotherapeutic sensitivity of osteosarcoma. Int J Clin Exp Pathol 7: 7114-7122, 2014

31. Moore N, Houghton J and Lyle S: Slow-cycling therapy-resistant cancer cells. Stem Cells Dev 21: 1822-1830, 2012.

32. Jungwirth U, Xanthos DN, Gojo J, Bytzek AK, Körner W, Heffeter P, Abramkin SA, Jakupec MA, Hartinger CG, Windberger $\mathrm{U}$, et al: Anticancer activity of methyl-substituted oxaliplatin analogs. Mol Pharmacol 81: 719-728, 2012. 
33. Shpilka T, Weidberg H, Pietrokovski S and Elazar Z: Atg8: An autophagy-related ubiquitin-like protein family. Genome Biol 12: 226, 2011.

34. Kabeya Y, Mizushima N, Ueno T, Yamamoto A, Kirisako T, Noda T, Kominami E, Ohsumi Y and Yoshimori T: LC3, a mammalian homologue of yeast Apg8p, is localized in autophagosome membranes after processing. EMBO J 19: 5720-5728, 2000.

35. Zhou YY, Li Y, Jiang WQ and Zhou LF: MAPK/JNK signalling: a potential autophagy regulation pathway. Biosci Rep 35: e0199, 2015.

36. Xiong $\mathrm{H}$, Zhang ZG, Tian XQ, Sun DF, Liang QC, Zhang YJ, Lu R, Chen YX and Fang JY: Inhibition of JAK1, 2/STAT3 signaling induces apoptosis, cell cycle arrest, and reduces tumor cell invasion in colorectal cancer cells. Neoplasia 10: 287-297, 2008.

37. Yuan L, Wei S, Wang J and Liu X: Isoorientin induces apoptosis and autophagy simultaneously by reactive oxygen species (ROS)-related p53, PI3K/Akt, JNK, and p38 signaling pathways in HepG2 cancer cells. J Agric Food Chem 62: 5390-5400, 2014

38. Jaber N, Dou Z, Chen JS, Catanzaro J, Jiang YP, Ballou LM, Selinger E, Ouyang X, Lin RZ, Zhang J and Zong WX: Class III $\mathrm{PI} 3 \mathrm{~K}$ Vps34 plays an essential role in autophagy and in heart and liver function. Proc Natl Acad Sci USA 109: 2003-2008, 2012.

39. Zhang DM, Liu JS, Deng LJ, Chen MF, Yiu A, Cao HH, Tian HY, Fung KP, Kurihara H, Pan JX and Ye WC: Arenobufagin, a natural bufadienolide from toad venom, induces apoptosis and autophagy in human hepatocellular carcinoma cells through inhibition of PI3K/Akt/mTOR pathway. Carcinogenesis 34: 1331-1342, 2013

40. Bai R, Ding T, Zhao J, Liu S, Zhang L, Lan X, Yu Y and Yin L: The effect of PI3K inhibitor LY294002 and gemcitabine hydrochloride combined with ionizing radiation on the formation of vasculogenic mimicry of Panc-1 cells in vitro and in vivo. Neoplasma 63: 80-92, 2016.

41. Li J, Yang D, Wang W, Piao S, Zhou J, Saiyin W, Zheng C, Sun H and Li Y: Inhibition of autophagy by 3-MA enhances IL-24induced apoptosis in human oral squamous cell carcinoma cells. J Exp Clin Cancer Res 34: 97, 2015.

42. Yamada H: Th17 cells in human rheumatoid arthritis. Nihon Rinsho Meneki Gakkai Kaishi 32: 249-255, 2009 (In Japanese).

43. Zou W and Restifo NP: T(H)17 cells in tumour immunity and immunotherapy. Nat Rev Immunol 10: 248-256, 2010.

44. Novatchkova M, Leibbrandt A, Werzowa J, Neubüser A and Eisenhaberemail F: The STIR-domain superfamily in signal transduction, development and immunity. Trends Biochem Sci 28: 226-229, 2003

45. Schraml BU, Hildner K, Ise W, Lee WL, Smith WA, Solomon B, Sahota G, Sim J, Mukasa R, Cemerski S, et al: The AP-1 transcription factor Batf controls $\mathrm{T}(\mathrm{H}) 17$ differentiation. Nature 460 : 405-409, 2009

46. Zhang X, Weng W, Xu W, Wang Y, Yu W, Tang X, Ma L, Pan Q, Wang J and Sun F: Prognostic significance of interleukin 17 in cancer: A meta-analysis. Int J Clin Exp Med 7: 3258-3269, 2014.

47. Zhang JP, Yan J, Xu J, Pang XH, Chen MS, Li L, Wu C, Li SP and Zheng L: Increased intratumoral IL-17-producing cells correlate with poor survival in hepatocellular carcinoma patients. J Hepatol 50: 980-989, 2009.

48. Kryczek I, Banerjee M, Cheng P, Vatan L, Szeliga W, Wei S, Huang E, Finlayson E, Simeone D, Welling TH, et al: Phenotype, distribution, generation, and functional and clinical relevance of Th17 cells in the human tumor environments. Blood 114: 1141-1149, 2009.
49. Hambright HG and Ghosh R: Autophagy: In the cROSshairs of cancer. Biochem Pharmacol (In Press).

50. Marin JJ, Lozano E and Perez MJ: Lack of mitochondrial DNA impairs chemical hypoxia-induced autophagy in liver tumor cells through ROS-AMPK-ULK1 signaling dysregulation independently of HIF-1 $\alpha$. Free Radic Biol Med 101: 71-84, 2016.

51. Weiland A, Roswall P, Hatzihristidis TC, Pietras K, Ostman A and Strell C: Fibroblast-dependependent regulation of the stem cell properties of cancer cells. Neoplasma 59: 719-727, 2012.

52. Amaravadi RK, Yu D, Lum JJ, Bui T, Christophorou MA, Evan GI, Thomas-Tikhonenko A and Thompson CB: Autophagy inhibition enhances therapy-induced apoptosis in a Myc-induced model of lymphoma. J Clin Invest 117: 326-336, 2007.

53. Yuan J, Zhang Y, Sheng Y, Fu X, Cheng H and Zhou R: MYBL2 guides autophagy suppressor VDAC2 in the developing ovary to inhibit autophagy through a complex of VDAC2-BECN1-BCL2L1 in mammals. Autophagy 11: 1081-1098, 2015.

54. Xu Y,LiZ, Yin Y,Lan H, Wang J, Zhao J, Feng J, Li Y and Zhang W: Ghrelin inhibits the differentiation of T helper 17 cells through mTOR/STAT3 signaling pathway. PLoS One 10: e0117081, 2015.

55. Li P, Shi J, He Q, Hu Q, Wang YY, Zhang LJ, Chan WT and Chen WX: Streptococcus pneumoniae Induces autophagy through the inhibition of the PI3K-I/Akt/mTOR pathway and ROS hypergeneration in A549 cells. PLoS One 10: e0122753, 2015.

56. Aveleira CA, Botelho M, Carmo-Silva S, Pascoal JF, Ferreira-Marques M, Nóbrega C, Cortes L, Valero J, Sousa-Ferreira L, Álvaro AR, et al: Neuropeptide Y stimulates autophagy in hypothalamic neurons. Proc Natl Acad Sci USA 112: E1642-E1651, 2015.

57. Wang P, Guo QS, Wang ZW and Qian HX: HBx induces HepG-2 cells autophagy through PI3K/Akt-mTOR pathway. Mol Cell Biochem 372: 161-168, 2013.

58. Huang F, Kao CY, Wachi S, Thai P, Ryu J and Wu R: Requirement for both JAK-mediated PI3K signaling and ACT1/TRAF6/TAK1-dependent NF-карpaB activation by IL-17A in enhancing cytokine expression in human airway epithelial cells. J Immunol 179: 6504-6513, 2007.

59. Sun X, Zhang J, Hou Z, Han Q, Zhang C and Tian Z: miR-146a is directly regulated by STAT3 in human hepatocellular carcinoma cells and involved in anti-tumor immune suppression. Cell Cycle 14: 243-252, 2015

60. Cross-Knorr S, Lu S, Perez K, Guevara S, Brilliant K, Pisano C, Quesenberry PJ, Resnick MB and Chatterjee D: RKIP phosphorylation and STAT3 activation is inhibited by oxaliplatin and camptothecin and are associated with poor prognosis in stage II colon cancer patients. BMC Cancer 13: 463, 2013.

61. Kim KW, Cho ML, Park MK, Yoon CH, Park SH, Lee SH and Kim HY: Increased interleukin-17 production via a phosphoinositide 3-kinase/Akt and nuclear factor kappaB-dependent pathway in patients with rheumatoid arthritis. Arthritis Res Ther 7: R139-R148, 2005.

62. Huang C, Yang G, Jiang T, Huang K, Cao J and Qiu Z: Effects of IL-6 and AG490 on regulation of Stat 3 signaling pathway and invasion of human pancreatic cancer cells in vitro. J Exp Clin Cancer Res 29: 51, 2010. 